BNL-97122-2012-IR

C-A/AP/\#454

Feb. 2012

\title{
Analytic approximate radiation effects due to Bremsstrahlung
}

\author{
I. Ben-Zvi
}

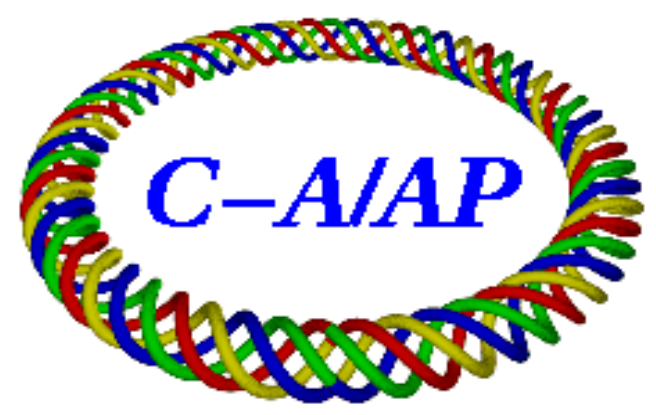

\section{Collider-Accelerator Department Brookhaven National Laboratory Upton, NY 11973}

Notice: This document has been authorized by employees of Brookhaven Science Associates, LLC under Contract No. DE-AC02-98CH10886 with the U.S. Department of En ergy. The United States Government retains a nonexclusive, paid-up, irrevocable, world-wide license to publish or reproduce the published form of this document, or allow others to do so, for United States Government purposes. 


\title{
Analytic Approximate Radiation Effects due to Bremsstrahlung
}

\author{
Ilan Ben-Zvi February 2012
}

\section{Introduction}

The purpose of this note is to provide analytic approximate expressions that can provide quick estimates of the various effects of the Bremsstrahlung radiation produced relatively low energy electrons, such as the dumping of the beam into the beam stop at the ERL or field emission in superconducting cavities. The purpose of this work is NOT to replace a dependable calculation or, better yet, a measurement under real conditions, but to provide a quick but approximate estimate for guidance purposes only. These effects include dose to personnel, ozone generation in the air volume exposed to the radiation, hydrogen generation in the beam dump water cooling system and radiation damage to near-by magnets. These expressions can be used for other purposes, but one should note that the electron beam energy range is limited. In these calculations the good range is from about $0.5 \mathrm{MeV}$ to $10 \mathrm{MeV}$.

To help in the application of this note, calculations are presented as a worked out example for the beam dump of the R\&D Energy Recovery Linac.

\section{Generation of the Bremsstrahlung}

The dumping of electrons with a kinetic energy $V$ and a current $\mathrm{I}_{\mathrm{e}}$ leads to electron beam power of $\mathrm{P}=\mathrm{VI}$, where $\mathrm{V}$ is the electron beam energy in $\mathrm{eV}$ and $\mathrm{I}_{\mathrm{e}}$ is the current in amperes.

The efficiency of converting electron energy into X-ray energy is

$\varepsilon=0.33^{*} 10^{-9 *} \mathrm{~V}^{*} \mathrm{Z} \quad$ reference $[1]$

where $\mathrm{V}$ is in $\mathrm{eV}, \mathrm{Z}$ is the atomic number of the material stopping the electrons (copper in the case of the beam dump).

This conversion factor is conservative, applicable for electrons below $0.5 \mathrm{MeV}$. For electrons of higher energy, between 0.5 to $4.6 \mathrm{MeV}$, the conversion factor is smaller by as much as $40 \%$.

Thus we get the power of the X-rays (in watts): 
$\mathrm{I}=\varepsilon \mathrm{VI}_{\mathrm{e}}=0.33^{*} 10^{-9 *} \mathrm{~V}^{2} * \mathrm{Z}^{*} \mathrm{I}_{\mathrm{e}}$

For the case of the ERL beam dump, $\mathrm{Z}=29, \mathrm{~V}=3 \times 10^{6} \mathrm{eV}$ and $\mathrm{I}_{\mathrm{e}}=0.33$ Amperes, which gives for the X-ray power $\mathrm{I}=19 \mathrm{~kW}$.

\begin{tabular}{|l|l|l|l|l|}
\hline Symbol & Parameter & Value & Units & Reference \\
\hline $\mathrm{Z}$ & Atomic number of copper & 29 & Atomic number & \\
\hline$\rho$ & Density of iron & 7800 & $\mathrm{~kg} / \mathrm{m}^{3}$ & \\
\hline$\rho$ & Density of concrete & 2300 & $\mathrm{~kg} / \mathrm{m}^{3}$ & \\
\hline$\rho$ & Density of air & $1.3 \times 10^{-6}$ & $\mathrm{~kg} / \mathrm{m}^{3}$ & \\
\hline$\mu^{\mathrm{a}} / \rho$ & Absorption coef. in water (body) & 0.003 & $\mathrm{~m}^{2} / \mathrm{kg}$ & {$[2,3]$} \\
\hline $\mathrm{r}$ & Rate of ozone generation & $6 \times 10^{17}$ & Molecules /Joule & {$[4]$} \\
\hline $\mathrm{h}$ & Hydrogen generation in water & $0.3 \times 10^{-3}$ & $\mathrm{cc} /$ Joule & \\
\hline
\end{tabular}

Table 1. Various useful parameters

At the energy of the ERL beam dump, $3 \mathrm{MeV}$, there is a significant forward beaming of the X-rays. This effect is considered later on in the calculation of the dose rate.

However, we briefly note that at $3 \mathrm{MeV}$ the half-angle $1 / \gamma$ is about 0.146 radians or 8.3 degrees. The enhancement can be quite large $\sim 200$. However, the opening angle of the beam may be increased by the divergence of the electron beam.

In the ERL beam dump the beam spread is enhanced by the beam optics to disperse the power deposition. The estimated spread of the ERL dump beam [5] is 10 to 15 degrees half-angle. This will reduce the above enhancement by at least a factor of 4 .

\section{Attenuation of the Bremsstrahlung X-rays}

The attenuation $\mathrm{A}$ of $\mathrm{X}$-rays by a given material is given by

$A=\exp (-\mu d)$

Where $\mu$ is the attenuation coefficient in $\mathrm{m}^{-1}$, and $\mathrm{d}$ is its thickness in $\mathrm{m}$.

In the following, with the single exception of calculating the X-ray absorption in water (representing a human body), the attenuation coefficients $\mu$ are total attenuation, which include various absorption AND scattering processes. The mechanism that represents scattered radiation making it out through the shielding is accounted for by the so called "Build-up Factor" discussed below.

It is remarkable that the total mass attenuation coefficients of light, disparate materials are very similar. Iron and lead are different at $0.1 \mathrm{MeV}$ and $100 \mathrm{MeV}$, but still fairly similar to the other materials at intermediate energies. 


\begin{tabular}{|l|l|l|l|l|l|l|}
\hline & Air & Water & Aluminum & Concrete & Iron & Lead \\
\hline $\mathbf{0 . 1} \mathbf{~ M e V}$ & 0.15 & 0.16 & 0.16 & 0.17 & 0.37 & $\sim 5$ \\
\hline $\mathbf{1 ~ M e V}$ & 0.06 & 0.07 & 0.06 & 0.06 & 0.06 & 0.07 \\
\hline $\mathbf{1 0 ~ M e V}$ & 0.015 & 0.022 & 0.023 & 0.023 & 0.03 & 0.05 \\
\hline $\mathbf{1 0 0} \mathbf{~ M e V}$ & 0.015 & 0.017 & 0.025 & 0.021 & 0.04 & 0.09 \\
\hline
\end{tabular}

Table 2. Mass attenuation coefficients for various materials at a few energies.

\section{Effect of the X-ray spectrum}

Given the dependence of the attenuation on the energy of the X-rays and the fact the Bremsstrahlung produces a continuum of energy, we should make a correction for the energy distribution of the Bremsstrahlung X-rays. We will make two simplifying assumptions for thick target Bremsstrahlung:

1) The energy spectrum is linearly decreasing with the X-ray energy, reaching zero at the electron energy.

2) The total attenuation as a function of X-ray energy is decreasing linearly with energy, with the numbers given at the table evaluated at the maximum energy of 3 $\mathrm{MeV}$.

Using these two assumptions and using a spectrum made of four bins of energy, we can get a somewhat better estimate for the attenuation of Bremsstrahlung radiation:

$A_{s}=\exp (-\mu \mathrm{d}) / 16+3 \exp (-4 \mu \mathrm{d} / 3) / 16+5 \exp (-2 \mu \mathrm{d}) / 16+7 \exp (-4 \mu \mathrm{d}) / 16$

For the value of $\mu$ we use the data given in the table above.

In the example of the ERL beam dump, we assume a first shield of 0.25 meters iron, followed by 1.28 meters regular concrete shield (at certain location and direction the shielding is much heavier). For the iron, if we assume that all the X-rays are at 3 $\mathrm{MeV}$ (excessively-conservative), we get an exponent of 7.8, and an attenuation factor of $4.1 \times 10^{-4}$, while the application of the spectral dependence gives an attenuation factor of $3.2 \times 10^{-5}$. For the 1.28 meter concrete the exponent is 5.9 , the $3 \mathrm{MeV} \mathrm{X-ray}$ attenuation is $2.8 \times 10^{-3}$, the spectral attenuation is $2.5 \times 10^{-4}$. Clearly a consideration of the spectral distribution of the Bremsstrahlung radiation makes a big difference in the estimate of the shield effectiveness.

\section{Build-up factor}

Following a large attenuation, a correction factor called "build-up factor" $\mathrm{k}$ has to be included. As mentioned above, this factor accounts for scattered radiation which contributes to the total mass attenuation coefficients but finds its way past the shielding. This factor for the case of an ERL beam dump, with beam generated by 3 $\mathrm{MeV}$ electrons, the shields are $25 \mathrm{~cm}$ iron and $128 \mathrm{~cm}$ outer shield of concrete is about 16, [6]. This correction takes into account that a pencil beam is attenuated by 
both absorption and scattering, but the scattered X-rays from one direction may make it back to another direction, thus reducing the effective attenuation.

Again, in the spirit of providing a quick and approximate estimate of the build-up factor, we use an expansion of the build-up factor into a power series in the exponent of the total attenuation [7] between the source and the observer. If we this exponent as $\mathrm{x}$, we have

$x=\sum_{n} \mu_{n} d_{n}$

where $\mathrm{n}$ sums over all layers of shields, with $\mu_{\mathrm{n}}$ being the attenuation coefficient for the material of the shield in the proper energy range and $d_{n}$ being the thickness of this shield. Then the build-up factor B can be written as

$$
B=1+\alpha x+\beta x^{2}+\gamma x^{3}
$$

The coefficients $\alpha, \beta$ and $\gamma$ are functions of both material and energy. Still, in the spirit of simplification, we will use the coefficients for iron as approximate values and select the energy from the following table:

$\begin{array}{lrrrr}\text { Energy (MeV) } & \mathbf{0 . 5} & \mathbf{1} & \mathbf{3} & \mathbf{1 0} \\ \boldsymbol{\alpha} & 0.992 & 0.955 & 0.553 & 0.34 \\ \boldsymbol{\beta} & 0.45 & 0.18 & 0.027 & -0.01 \\ \gamma & 0.004 & -0.0026 & -0.0004 & 0.003\end{array}$

Table 3. Coefficients for power expansion of the build-up factor as function of energy.

The build-up factor for the ERL beam dump can be obtained using the above expression and the shields described in the previous subsection (.125 meter iron and 1.28 meter concrete). The expression yields $\mathrm{B}=34$ at $3 \mathrm{MeV}$, reasonably close to the factor of 16 provided in Ref [6].

\section{Dose rate calculation}

Initially we assume that the X-ray radiation is isotropic. The relativistic angular distribution will be then factored in.

The radiation flux in Watt $/ \mathrm{m}^{2}$ at a distance $\mathrm{R}(\mathrm{m})$ from the dump is

$\mathrm{F}=\mathrm{I} \mathrm{A}_{1} \mathrm{~A}_{2} \mathrm{~B} /\left(4 \pi \mathrm{R}^{2}\right)$,

where $A_{n}$ is an attenuation coefficient in material $n$.

If this flux encounters water (a human body is mostly water) the energy deposition $D$ in watt per kilogram is 
$\mathrm{D}=\mathrm{F}\left(\mu^{\mathrm{a}}{ }_{\text {water }} / \rho_{\text {water }}\right)$

$\rho$ is the density of the material in $\mathrm{kg} / \mathrm{m}^{3}$.

Where $\mu^{\mathrm{a}}$ water is the absorption of the X-rays in water (the absorption is a partial process to attenuations). $\mu^{\mathrm{a}}$ water $/ \rho_{\text {water }}$ is fairly constant (independent of energy) in the X-ray energy range of $0.5 \mathrm{MeV}$ to $5 \mathrm{MeV}$, at $\mu^{\mathrm{a}}{ }_{\text {water }} / \rho_{\text {water }} \sim 0.003$

The dose rate $\mathrm{G}$ in Greys/second is the energy deposited in Watt per kg, so

$G=D$,

and in Rads/second (100 rads per Grey)

$\mathrm{R}=100 \mathrm{G}=100 \mu^{\mathrm{a}}{ }_{\text {water }} / \rho_{\text {water }} \mathrm{IA}_{1} \mathrm{~A}_{2} \mathrm{~B} /\left(4 \pi \mathrm{R}^{2}\right)$

Substituting the value for $\mu^{\mathrm{a}}$ water $/ \rho_{\text {water }}=0.003$ we get

$\mathrm{R}=0.3 \mathrm{IA}_{1} \mathrm{~A}_{2} \mathrm{~B} /\left(4 \pi \mathrm{R}^{2}\right)=0.024 \mathrm{IA}_{1} \mathrm{~A}_{2} \mathrm{~B} / \mathrm{R}^{2}$

Further substitution of the expressions for the X-ray power $\mathrm{I}=\varepsilon \mathrm{I} \mathrm{I}_{\mathrm{e}} * \mathrm{~V}$ and

$\varepsilon=0.33^{*} 10^{-9 *} \mathrm{VZ}$, or $\mathrm{I}=0.33^{*} 10^{-9 *} \mathrm{I} \mathrm{e} \mathrm{V}^{2} \mathrm{Z}$ gives us

$\mathrm{R}(\mathrm{Rem} / \mathrm{s})=8^{*} 10^{-12 *} \mathrm{Ie}_{\mathrm{e}} \mathrm{V}^{2} \mathrm{Z} \mathrm{IA}_{1} \mathrm{~A}_{2} \mathrm{~B} / \mathrm{R}^{2}$

Now let us use the $\mathrm{Z}=29$ of copper and build-up factor $\mathrm{B} \sim 34$.

Let us also use a current of 0.33 amperes, voltage of $3 \times 10^{6}$ volts, attenuation factor of $A_{1}=0.0024$ for $0.125 \mathrm{~m}$ iron, $A_{2}=2.5 \times 10^{-4}$ for $1.28 \mathrm{~m}$ of concrete, and a distance $\mathrm{R}$ $=5 \mathrm{~m}$, then we get $\mathrm{R}=2000 \mathrm{mR} / \mathrm{hr}$.

\section{Angular distribution for relativistic electrons}

The motion of the source (the electrons) modifies the angular distribution quite significantly, and that must be taken into account.

We will use the following expression for the angular distribution [8]

$$
\phi=\left[\gamma\left(1-\sqrt{1-\gamma^{-2}} \cos (\theta)\right)\right]^{-4}
$$

where $\phi$ should multiply the dose rate $\mathrm{R}$ in order to account for the relativistic beaming of the Bremsstrahlung radiation in a direction $\theta$ relative to the motion of the electron. At $3 \mathrm{MeV}$ the relativistic factor $\gamma$ is 6.44, and at an angle of $\pi / 2$ to the beam the correction factor is $\phi=0.00058$. Then the final angle corrected dose rate is

$\mathrm{R}^{*} \phi=1 \mathrm{mRad} /$ hour. This is the point where one expects the highest dose rate outside but near the concrete shield. 
As was already mentioned, the angular spread of the beam entering the beam dump significantly reduces the enhancement in the forward direction, but does not affect larger angles such as 90 degrees to the beam.

\section{Ozone generation}

The rate of generation of ozone molecules is [4] 0.1 molecule per eV, or $r=6^{*} 10^{17}$ molecules per Joule of deposited energy. The acceptable limit [9] is $0.1 \mathrm{ppm}$ of ozone in the air.

The concentration of the ozone depends on the X-ray power as well as the volume of air over which it is distributed, so the size of the participating volume is important. We will assume a small source of radiation that expands spherically into $4 \pi$, but if the radiation expands into any slice of this volume as limited by absorbing walls, the result does not depend on the angular opening of the slice, only on the maximum radius of expansion.

The absorption of the X-rays will be assumed to be independent of the distance traveled in air, as the absorption is small. Thus

and

$$
\frac{d I}{d x}=\mu I
$$

$$
\mu=\frac{\mu}{\rho} \rho
$$

and the number $\mathrm{N}$ of ozone molecules per unit distance traveled by the $\mathrm{X}$-rays is

$$
\frac{d N}{d x}=r \mu I
$$

This rate is independent of the area over which the X-ray power is distributed. However, the concentration does depend on the area, since the number of air molecules per unit length along the direction of propagation depends on the area included. Thus it is natural that spreading the X-ray power over a larger volume will generate the same amount of ozone molecules, but a smaller concentration.

Let the number of air molecules per unit distance be $\mathrm{N}_{\mathrm{a}}$, and assuming a molecular weight of 28 and density $\rho$ for air, we have

$$
\frac{d N_{a}}{d x}=6 \times 10^{23} \rho A / 28
$$

Thus the concentration c for a unit distance is 


$$
\begin{gathered}
c=I \frac{r \mu}{\rho} \frac{\int d x}{2.1 \times 10^{22} \int 4 \pi x^{2} d x} \\
c=\operatorname{Ir} \frac{\mu}{\rho} \frac{1}{7.9 \times 10^{23}}\left[\frac{x_{2}-x_{1}}{x_{2}^{3}-x_{1}^{3}}\right]
\end{gathered}
$$

Also, assume that $\mathrm{X}_{2}>>\mathrm{x}_{1}$

$$
c=1.26 \times 10^{-24} \operatorname{Ir} \frac{\mu}{\rho} \frac{1}{x_{2}{ }^{2}}
$$

Now let us substitute values for $\mathrm{r}$ and $\mu / \rho$ of air, then, with $\mathrm{x}$ in $\mathrm{cm}$,

$$
c=2.3 \times 10^{-14} \frac{I}{x_{2}{ }^{2}}
$$

For the above example of $\mathrm{I}=19333$ Watts and $\mathrm{x}_{2}$ of $10 \mathrm{~m}$, we get an ozone concentration buildup per second of $\mathrm{c}=4.4 \times 10^{-10}$, or $1.6 \mathrm{ppm}$ per hour for CW operation at $1 \mathrm{MW}$ beam power with no shielding. Now, assuming a shield of iron $0.25 \mathrm{~m}$ thick next to the dump, the ozone production rate goes down to about $10^{-4}$ ppm per hour, a totally negligible rate.

\section{Hydrogen generation in water}

Let the integrated radiation flux right next to the beam dump be I. Assume this flux is passing through $\mathrm{w} \mathrm{cm}$ of water. The energy deposition in the water does not depend on the area of the radiation, just the thickness of the water envelope. Then the energy deposition rate in the water is

$\mathrm{D}=\mathrm{wI}\left(\mu_{\text {water }} / \rho_{\text {water }}\right) \rho_{\text {water }}$

For $\mathrm{w}=0.03 \mathrm{~m}$, we get $\mathrm{D}=0.03^{*} 19333^{*} 0.003^{*} 1000 \sim 1700$ watts, which will generate $\mathrm{hD} c c$ hydrogen per second. Since $\mathrm{h}=0.3 \times 10^{-3}[10,11]$, the hydrogen generation rate for a CW $1 \mathrm{MW}$ beam of $3 \mathrm{MeV}$ in a $3 \mathrm{~cm}$ water envelope is $\sim 0.5 \mathrm{cc} / \mathrm{second}$, or 1900 cc/hour.

The forward directed radiation is much worse for two reasons. One, the beaming of the forward directed radiation, the second reason is the large volume of water in the forward direction. This volume has about $24000 \mathrm{CC}$ of water, and let us approximate the length to be $0.1 \mathrm{~m}$. Further assume that all the power of 19000 watt is going through this water volume, then we about triple the hydrogen generation rate, call it 
5.6 liter per hour. This is not a significant amount of hydrogen, and it can be vented safely (no radio-isotopes).

\section{Material damage in dump's immediate vicinity}

The radius of the dump stainless steel envelope is $r_{d}=0.21 \mathrm{~m}$. Assume the electrons are stopped over a band of $L_{d}=0.40 \mathrm{~m}$ in length. Then the flux (neglecting possible beaming) is (in watts per square $\mathrm{m}$ )

$$
F_{d}=\frac{I}{2 \pi L_{d} r_{d}}
$$

and the radiation dose rate in Rad/second for a material with mass attenuation coefficient $\mu / \rho$ and density $\rho$ will be

$$
R=10^{2} F_{d} \mu / \rho=10^{2} \frac{I \mu / \rho}{2 \pi L_{d} r_{d}}
$$

Assume I=19000 W, $\mu / \rho \sim 0.003$, then R 10800 Rad/second, or $38 \mathrm{MRad} /$ hour. For magnets at a distance of 1 meter, the dose is reduced by a factor of $25\left(\mathrm{r}_{\mathrm{d}}\right.$ five times larger) to $1.5 \mathrm{Mrad} / \mathrm{hour}$.

According to reference [12], the radiation limit of various materials is as follows:

Thermalastic resin -1 GRad

PVC - 0.1 GRad

Thermoplastic polyethylene - $0.1 \mathrm{GRad}$

Teflon, KEL F, - 0.001 GRad

Polyethylene Oxide (PPO) - 2 GRad

Mylar - 0.5 GRad

Kapton - >2 GRad

Assuming a material radiation damage limit of $1 \mathrm{GRad}$, damage can result in 600 hours of operation.

This dose is sufficiently high to worry about long term effects on epoxy in magnets, so some shielding will have to be introduced before full power operations commence. If we assume $25 \mathrm{~cm}$ of iron shielding between the dump and the magnets the reduction in the radiation is by a factor of 0.0004 , to $600 \mathrm{Rad} / \mathrm{hour}$, a safe number, allowing over 1 million hours of operation before damage.

\section{Comparison with MCNPX}

Kin Yip did a calculation of the beam dump [13], using the MCNPX code [14]. Running the electrons at $3 \mathrm{MeV}$, beam power of $1 \mathrm{MW}$, he gets hydrogen generation of 4.8 Littre/hour, which is close to the calculation done above (5.6 Littre/hour). 
The calculated dose at about 90 degrees to the beam and at a distance of about 5 meters the code gives about $0.07 \mathrm{mRad} /$ hour, which is about 14 times smaller than the estimate in our worked out example above (1 mRad/hour). Thus our estimate is conservative, by about an order of magnitude.

\section{Acknowledgements}

Many thanks go to Dana Beavis, Mike Blaskiewicz, Ray Karol, Ed Lessard, and Kin Yip for help on various aspects of this work.

\section{References}

[1] The Atomic Nucleus, R. D. Evans, McGraw-Hill 1955, p.619

[2] Ibid, p.714-715.

[3] NIST http://www.nist.gov/pml/data/xraycoef/index.cfm

[4] NCRP Report No. 144, Radiation Protection for Particle Accelerator Facilities, issued December 31, 2003. Section 6.4 Radiolysis in Water and Air, Table 6.8.

[5] D. Kayran, private communication

[6] Calculation by courtesy of Edward Lessard, performed with MicroShield v5.05 (5.05-00047) Grove Engineering, Inc.

[7] G. E. Chabot, K. W. Skrable, H. L. Wedlick, Notes on Buildup Factors and Utilization of a Power Function Analytical Representation of the Buildup Factor, Health Physics, Pergamon Press 1971. Vol. 21 (Sept.), pp. 471-474. Printed in Northern Ireland

[8] Problem Book in Relativity and Gravitation, A.P. Lightman, W.H. Press, R.H.

Price, S.A. Teukolsky, Princeton University Press, Princeton NJ 1979, page 213

[9] American Conference of Government Industrial Hygienists (ACGIH) 2006 [10] SLAC-TN-67-29, D.R. Waltz and E.J. SEPPI, Radiolysis and Hydrogen Evolution in the A-Beam Dump Radioactive Water System, October 1967. http://www.slac.stanford.edu/cgi-wrap/getdoc/slac-tn-67-029.pdf [11] CEBAF-TN-0162, G. Stapleton, Radiolysis and Radioactivation of Beam Dum Cooling Water, August 14,1989. http://tnweb.jlab.org/tn/1989/89-162.pdf [12] Effects of radiation on materials and components, M.H. Van de Voorde, CERN 70-05

[13] Kin Yip, private communication

[14] http://mcnpx.lanl.gov 